\title{
A High-Speed Conditional Carry Select (CCS) Adder Circuit with a Successively Incremented Carry Number Block (SICNB) Structure for Low-Voltage VLSI Implementation
}

\author{
Yen-Mou Huang and James B. Kuo, Fellow, IEEE
}

\begin{abstract}
This paper reports a conditional carry select (CCS) adder circuit with a successively-incremented-carry-number block (SICNB) structure for low-voltage VLSI implementation. Owing to the successively-incremented-carry-number block (SICNB) structure, the new 16-bit SICNB CCS adder provides a $37 \%$ faster speed as compared to the conventional conditional carry select adder based on the SPICE results.
\end{abstract}

Index Terms-Carry select adders, high-speed, low-voltage, multipliers.

\section{INTRODUCTION}

$\mathbf{I}$ $\mathrm{N}$ A very large scale integration (VLSI) CPU, the adder is an important circuit. The speed performance of a CPU is predominantly determined by its adder circuit. Various adder circuits with improved speed performance have been reported [1]-[8]. Among them, the conditional carry select (CCS) adder [1] has a superior speed performance. As shown in Fig. 1, in a 16-bit CCS adder, the key circuit is the carry select circuit [1]. The principle of the carry select circuit is briefly described here. The carry select circuit is used to process the propagate and generate signals produced by the half adders to generate the carry signals. The function of the carry select circuit is

$$
C_{i}=G_{i}+C_{i-1} \cdot P_{i}, \quad \text { for } i=1 \sim n
$$

where $n$ is the bit number. $G_{i}$ and $P_{i}$ are the generate and propagate signals $\left(G_{i}=X_{i} \cdot Y_{i}, P_{i}=X_{i}+Y_{i}\right)$ produced from two inputs $\left(X_{i}, Y_{i}\right)$ to the half adder. From the above equation, if $C_{i-1}=0$, then $C_{i}=G_{i}$. If $C_{i-1}=1$, then $C_{i}=P_{i}$. Therefore, the carry signal can be reorganized as

$$
C_{i}=\overline{C_{i-1}} \cdot G_{i}+C_{i-1} \cdot P_{i} .
$$

As shown in Fig. 1, the 16-bit conditional carry select circuit is group into four carry blocks. In each carry block, there are

Manuscript received May 1999; revised May 2000. This work was supported by the R.O.C. National Science Council under Contract 88-2215-E002-033 and Contract 88-2622-E002-028. This paper was recommended by Associate Editor Y. Leblebici.

Y.-M. Huang was with the Department of Electrical Engineering, National Taiwan University, Sec. 4, Taipei, Taiwan 106. He is now with VIA Technologies, Hsin-Tien, Taipei 231, Taiwan.

J. B. Kuo is with the Department of Electrical Engineering, National Taiwan University, Sec. 4, Taipei, Taiwan 106 (e-mail: jbkuo@cc.ee.ntu.edu.tw).

Publisher Item Identifier S 1057-7130(00)09323-X four multiplexers (MUX) controlled by $C_{i-1}$ to produce four output carry signals $C_{i}, C_{i+1}, C_{i+2}, C_{i+3}$-the carry number of this conditional carry select circuit is four

$$
\begin{array}{r}
C_{i}=\overline{C_{j}} \cdot C_{i}\left(\text { if } C_{j}=0\right)+C_{j} \cdot C_{i}\left(\text { if } C_{j}=1\right) \\
i=j+1 \sim j+4 ; j=-1,3,7,11 .
\end{array}
$$

For example, in the first block, considering $C_{0}$, if $C_{\mathrm{in}}=1$, the output carry signal

$$
C_{0}=\overline{C_{\mathrm{in}}} \cdot G_{o}+C_{\mathrm{in}} \cdot P_{0}=P_{0} \equiv C_{0}\left(\text { if } C_{\mathrm{in}}=1\right) .
$$

If

$$
C_{\mathrm{in}}=0, \quad C_{0}=G_{0} \equiv C_{0}\left(\text { if } C_{\text {in }}=0\right) .
$$

As for $C_{1}$, if $C_{\text {in }}=1$

$$
\begin{aligned}
C_{1} & =\overline{C_{0}} \cdot G_{1}+C_{0} \cdot P_{1} \\
& =\overline{P_{0}} \cdot G_{1}+P_{0} \cdot P_{1} \equiv C_{1}\left(\text { if } C_{\text {in }}=1\right) .
\end{aligned}
$$

If $C_{\text {in }}=0$

$$
C_{1}=\overline{G_{0}} \cdot G_{1}+G_{\circ} \cdot P_{1} \equiv C_{1}\left(\text { if } C_{\text {in }}=0\right) .
$$

As for $C_{2}$, if $C_{\text {in }}=1$

$$
\begin{aligned}
C_{2} & =\overline{C_{1}} \cdot G_{2}+C_{1} \cdot P_{2} \\
& =\overline{\overline{P_{0}}} \cdot G_{1}+P_{0} \cdot P_{1} \cdot G_{2}+\left(\overline{P_{0}} \cdot G_{1}+P_{0} \cdot P_{1}\right) \cdot P_{2} \\
& \equiv C_{2}\left(\text { if } C_{\text {in }}=1\right) .
\end{aligned}
$$

$$
\begin{aligned}
& \text { If } C_{\mathrm{in}}=0 \\
& C_{2}=\overline{\overline{G_{0}} \cdot G_{1}+G_{0} \cdot P_{1}} \cdot G_{2}+\left(\overline{G_{0}} \cdot G_{1}+G_{0} \cdot P_{1}\right) \cdot P_{2} \\
& \equiv C_{2}\left(\text { if } C_{\text {in }}=0\right) \text {. }
\end{aligned}
$$

As for $C_{3}$, if $C_{\mathrm{in}}=1$,

$$
\begin{aligned}
& C_{3}=\overline{C_{2}} \cdot G_{3}+C_{2} \cdot P_{3} \\
& =\overline{\overline{P_{0}} \cdot G_{1}+P_{0} \cdot P_{1}} \cdot G_{2}+\left(\overline{P_{0}} \cdot G_{1}+P_{0} \cdot P_{1}\right) \cdot P_{2} \cdot G_{3} \\
& +\left(\overline{\overline{P_{0}}} \cdot G_{1}+P_{0} \cdot P_{1} \cdot G_{2}\right. \\
& \left.+\left(\overline{P_{0}} \cdot G_{1}+P_{0} \cdot P_{1}\right) \cdot P_{2}\right) \cdot P_{3} \\
& \equiv C_{3}\left(\text { if } C_{\text {in }}=1\right) \text {. }
\end{aligned}
$$




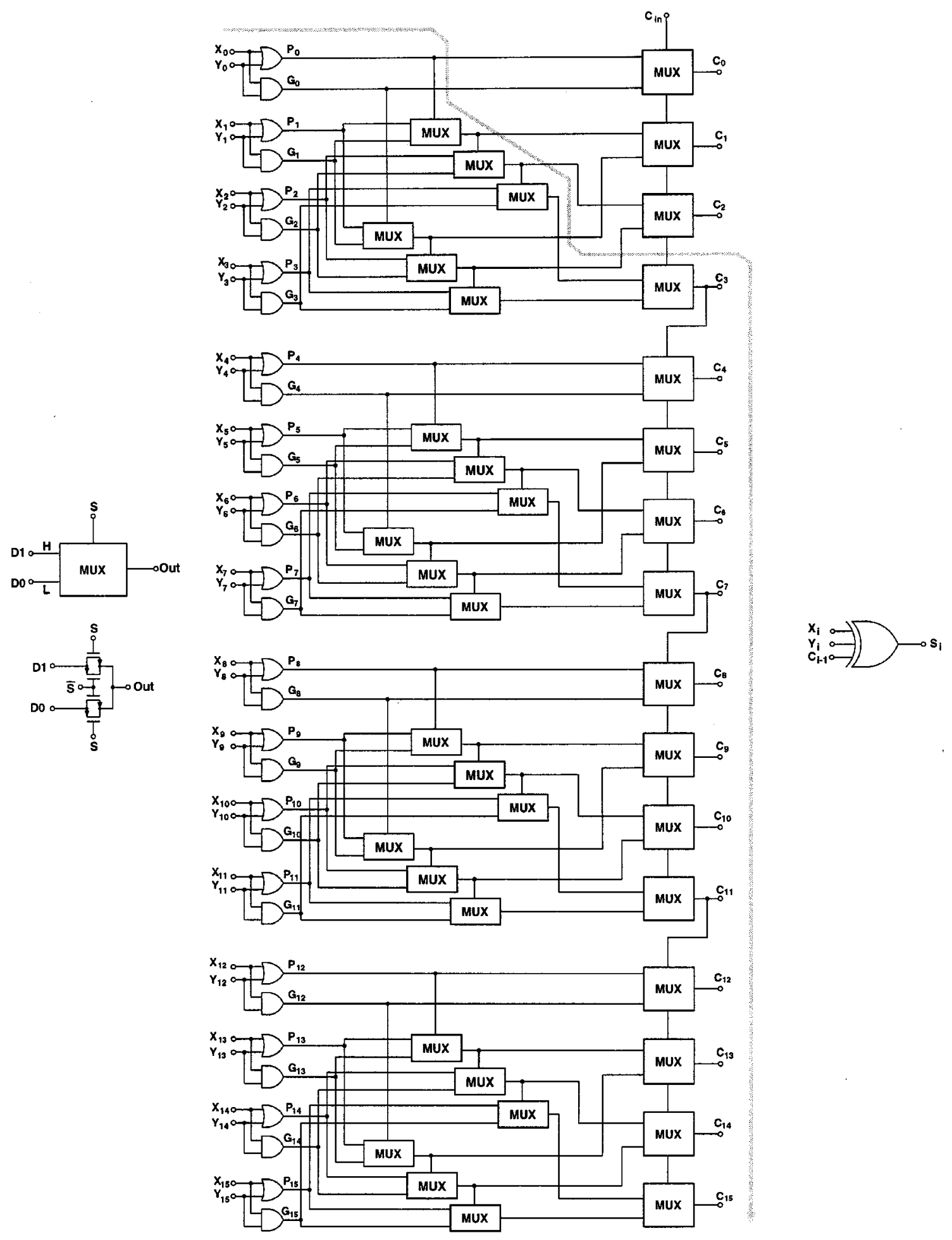

Fig. 1. Block diagram of a 16-bit CCS circuit.

$$
\begin{aligned}
& \text { If } C_{\mathrm{in}}=0 \\
& C_{3}=\overline{\overline{G_{0}} \cdot G_{1}+G_{0} \cdot P_{1}} \cdot G_{2}+\left(\overline{G_{0}} \cdot G_{1}+G_{0} \cdot P_{1}\right) \cdot P_{2} \cdot G_{3} \\
& +\left(\overline{\overline{G_{0}}} \cdot G_{1}+G_{0} \cdot P_{1} \cdot G_{2}\right. \\
& \left.+\left(\overline{G_{0}} \cdot G_{1}+G_{0} \cdot P_{1}\right) \cdot P_{2}\right) \cdot P_{3} \\
& \equiv C_{3}\left(\text { if } C_{\text {in }}=0\right) \text {. }
\end{aligned}
$$

In summary, there are four carry blocks in the 16-bit conditional carry select circuit. Each carry block produces four output carry signals. In the first block, with the multiplexer control signal $C_{\mathrm{in}}$, four output carry signals $\left(C_{0}, C_{1}, C_{2}, C_{3}\right)$ are produced. In the second block, with the multiplexer control signal $C_{3}$, four output carry signals $\left(C_{4}, C_{5}, C_{6}, C_{7}\right)$ are generated. In the third block, with the multiplexer control signal $C_{7}$, four 


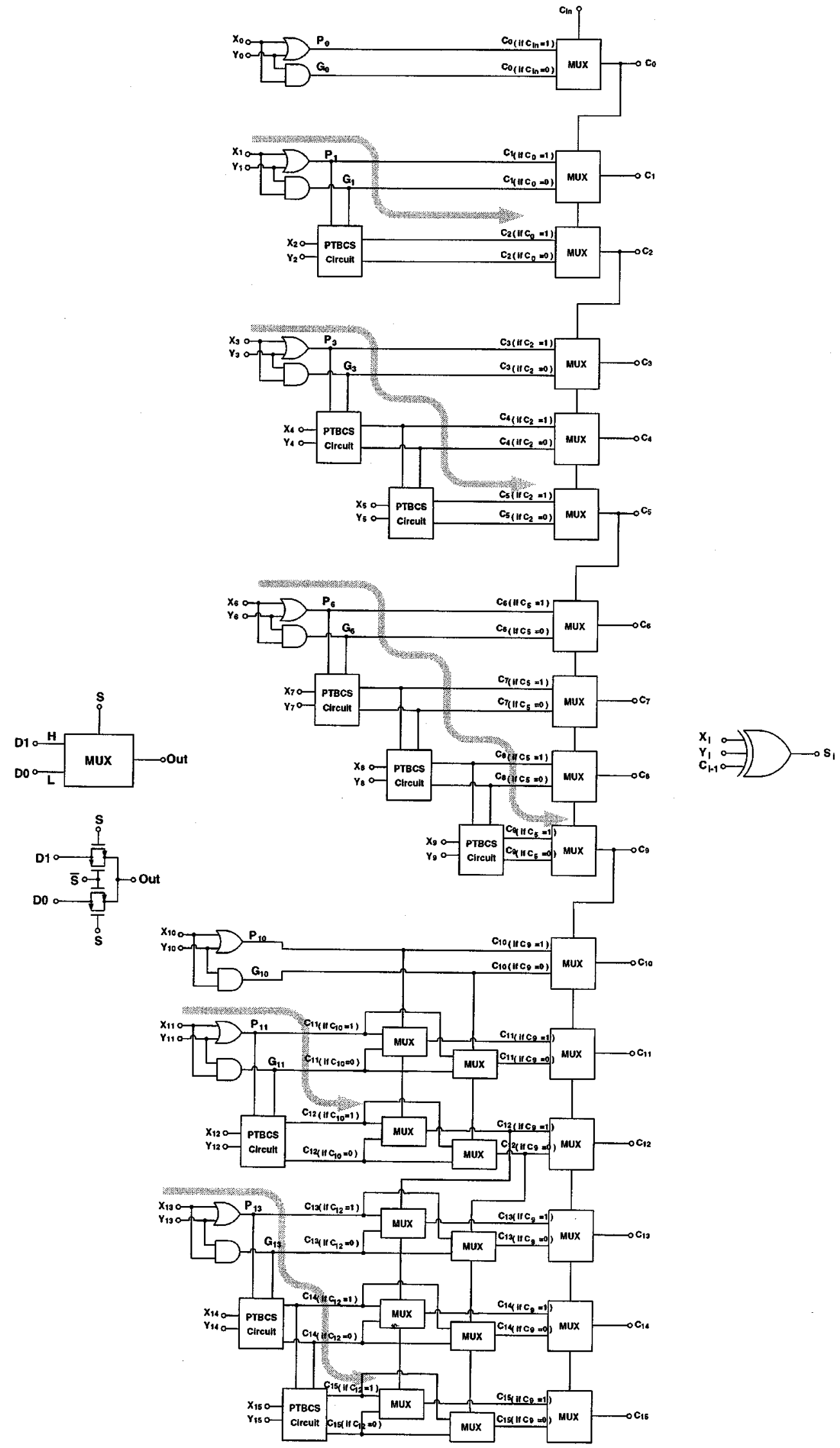

Fig. 2. Block diagram of the 16-bit SICNB structure CCS circuit.

output carry signals $\left(C_{8}, C_{9}, C_{10}, C_{11}\right)$ are produced. With $\left(C_{12}, C_{13}, C_{14}, C_{15}\right)$ are generated. The longest critical path is the multiplexer control signal $C_{11}$, four output carry signals from $X_{0}$ to $C_{15}$, which occurs at inputs $\left(X_{0} \sim X_{15}\right)=(1,0 \sim$ 
$0) ;\left(Y_{0} \sim Y_{15}\right)=(1 \sim 1)$, which involves gate delays of eight stages - one stage of $P_{0}$ or $G_{0}$, three stages of multiplexers, and four stages of carry.

\section{SuCCESSIVEly INCREMENTED CARRY Number Block (SICNB) CARRY SELECT ADDER}

Fig. 2 shows the 16-bit SICNB CCS circuit. As shown in the figure, five carry blocks, as in the conditional carry select circuit, have been used. Different from the conditional carry select circuit, the carry number in each carry block is not always four-not uniform. Instead, in the first carry block, only one output carry signal $C_{0}$ is produced

$$
\begin{gathered}
C_{i}=\overline{C_{j}} \cdot C_{i}\left(\text { if } C_{j}=0\right)+C_{j} \cdot C_{i}\left(\text { if } C_{j}=1\right) \\
j=-1, i=j+1 .
\end{gathered}
$$

In the second carry block, two output carry signals $C_{1}$ and $C_{2}$ are generated.

$$
\begin{gathered}
C_{i}=\overline{C_{j}} \cdot C_{i}\left(\text { if } C_{j}=0\right)+C_{j} \cdot C_{i}\left(\text { if } C_{j}=1\right) \\
j=0, i=j+1, j+2 .
\end{gathered}
$$

In the third carry block, three output carry signals $C_{3}-C_{5}$ are available

$$
\begin{gathered}
C_{i}=\overline{C_{j}} \cdot C_{i}\left(\text { if } C_{j}=0\right)+C_{j} \cdot C_{i}\left(\text { if } C_{j}=1\right) ; \\
j=2, i=j+1 \sim j+3 .
\end{gathered}
$$

In the fourth carry block, four output carry signals $C_{6}-C_{9}$ are generated.

$$
\begin{gathered}
C_{i}=\overline{C_{j}} \cdot C_{i}\left(\text { if } C_{j}=0\right)+C_{j} \cdot C_{i}\left(\text { if } C_{j}=1\right) \\
j=5, i=j+1 \sim j+4 .
\end{gathered}
$$

In the fifth carry block, six output carry signals $C_{10}-C_{15}$ are produced

$$
\begin{gathered}
C_{i}=\overline{C_{j}} \cdot C_{i}\left(\text { if } C_{j}=0\right)+C_{j} \cdot C_{i}\left(\text { if } C_{j}=1\right) \\
j=9, i=j+1 \sim j+6 .
\end{gathered}
$$

As shown in the figure, in the SICNB CCS adder, the number of output carry signals produced in each block is successively incremented from the first block to the last block.

The longest critical path is from $X_{0}$ to $C_{15}$, which occurs at inputs $\left(X_{0} \sim X_{15}\right)=(1,0 \sim 0) ;\left(Y_{0} \sim Y_{15}\right)=(1 \sim 1)$, which involves gate delays of six stages - one stage of $P_{0}$ or $G_{0}$ and five stages of multiplexers. Compared to the conventional conditional carry select circuit, the longest critical path is two stages shorter.

Fig. 3 shows the 1-bit pass-transistor-based carry select (PTBCS) circuit [4] used in the SICNB CCS adder of Fig. 2. Instead of $P_{i}$ in the conventional Manchester CLA circuit, $C_{i-1}$ is used as the control signal in the PTBCS circuit, as shown in Fig. 3. In addition, pass-transistor logic has been used to replace the multiplexer structure. The operation principle is described here. When $C_{i-1}=1$, the carry signal $\left(C_{i}=G_{i}+C_{i-1} \cdot P_{i}\right)$ becomes $C_{i}=P_{i}$, hence $P_{i}$ is used as the input to a transmission gate controlled by $C_{i-1}$. When $C_{i-1}=0$, the transmission gate is off. Under this situation, the circuit is similar to a $\overline{G_{i}}$-controlled inverter (when $\overline{C_{i-1}}$ is high; hence, the NMOS device $M N A$ is on). Therefore, $C_{i}=G_{i}$. Similarly

$$
\overline{C_{i}}=\overline{G_{i}} \cdot\left(\overline{C_{i-1}}+\overline{P_{i}}\right)=\overline{C_{i-1}} \cdot \overline{G_{i}}+\overline{G_{i}} \cdot \overline{P_{i}} .
$$

Since

$$
\overline{G_{i}} \cdot \overline{P_{i}}=\left(\overline{X_{i}}+\overline{Y_{i}}\right)\left(\overline{X_{i}} \cdot \overline{Y_{i}}\right)=\overline{X_{i}} \cdot \overline{Y_{i}}=\overline{P_{i}}
$$

therefore, $\overline{C_{i}}=\overline{P_{i}}+\overline{C_{i-1}} \cdot \overline{G_{i}}$. Similarly, $\overline{C_{i-1}}$ is used as the control signal. When $\overline{C_{i-1}}$ is high, $\overline{C_{i}}=\overline{P_{i}}+\overline{G_{i}}=\overline{G_{i}}$. Therefore, $\overline{G_{i}}$ can be used as an input to the transmission gate controlled by $C_{i-1}$. When $\overline{C_{i-1}}$ is low, the transmission gate is off. Under this situation, the above circuit becomes a $P_{i}$-controlled inverter (when $C_{i-1}$ is high, the nMOS device $M N B$ turns on), $\overline{C_{i}}=\overline{P_{i}}$. Using the pass-transistor logic in the PTBCS circuit, there are advantages: the output of one stage is connected to the gate of the next stage instead of the source/drain in the conventional Manchester CLA circuit. Therefore, no long serial critical path is involved in the circuit, which is especially useful for low-voltage operation.

\section{RESULTS}

In order to verify the effectiveness of the new adder, a 16-bit SICNB CCS adder has been designed based on a $0.8-\mu \mathrm{m}$ CMOS technology. The aspect ratio of the nMOS and the pMOS devices in the test chip is $8 \mu \mathrm{m} / 0.8 \mu \mathrm{m}$ and $16 \mu \mathrm{m} / 0.8$ $\mu \mathrm{m}$, respectively. Fig. 4 shows the transient waveforms of the 8th and the 16th bit carry signals $\left(C_{7}, C_{15}\right)$ of the 16-bit SICNB CCS adder at $V_{d d}$ of $1.5 \mathrm{~V}$ with two inputs to be added as $\left(X_{0} \sim X_{15}\right)=(1,0 \sim 0) ;\left(Y_{0} \sim Y_{15}\right)=(1 \sim 1)$, based on SPICE simulation results. As shown in the figure, the propagation delay from the input $X_{0}$ to the 16th bit carry out signal $\left(C_{15 A}\right)$ is $14.56 \mathrm{~ns}$, which is $37 \%$ faster as compared to the conventional CCS adder without SICNB structure. Fig. 5 shows the propagation delay of the 16th bit carry signal $\left(C_{15}\right)$ versus the power supply voltage used in the SICNB CCS adder. As shown in the figure, the SICNB CCS adder has a consistent improvement over the conventional CCS adder regardless of the power supply voltage. Table I shows the power consumption of a 16-bit adder implemented by conventional CCS technique and the SICNB CCS technique. As shown in the table, using the SICNB CCS technique, the power consumption increases mildly as compared to the adder implemented by the conventional CCS technique.

CCS adders have been well known for their advantages in high speed as compared to Manchester adders. The SICNB CCS adder presented in this paper can provide an even higher speed performance as compared to the CCS adder. The applicability of the SICNB CCS adder is discussed here. In this paper, the 


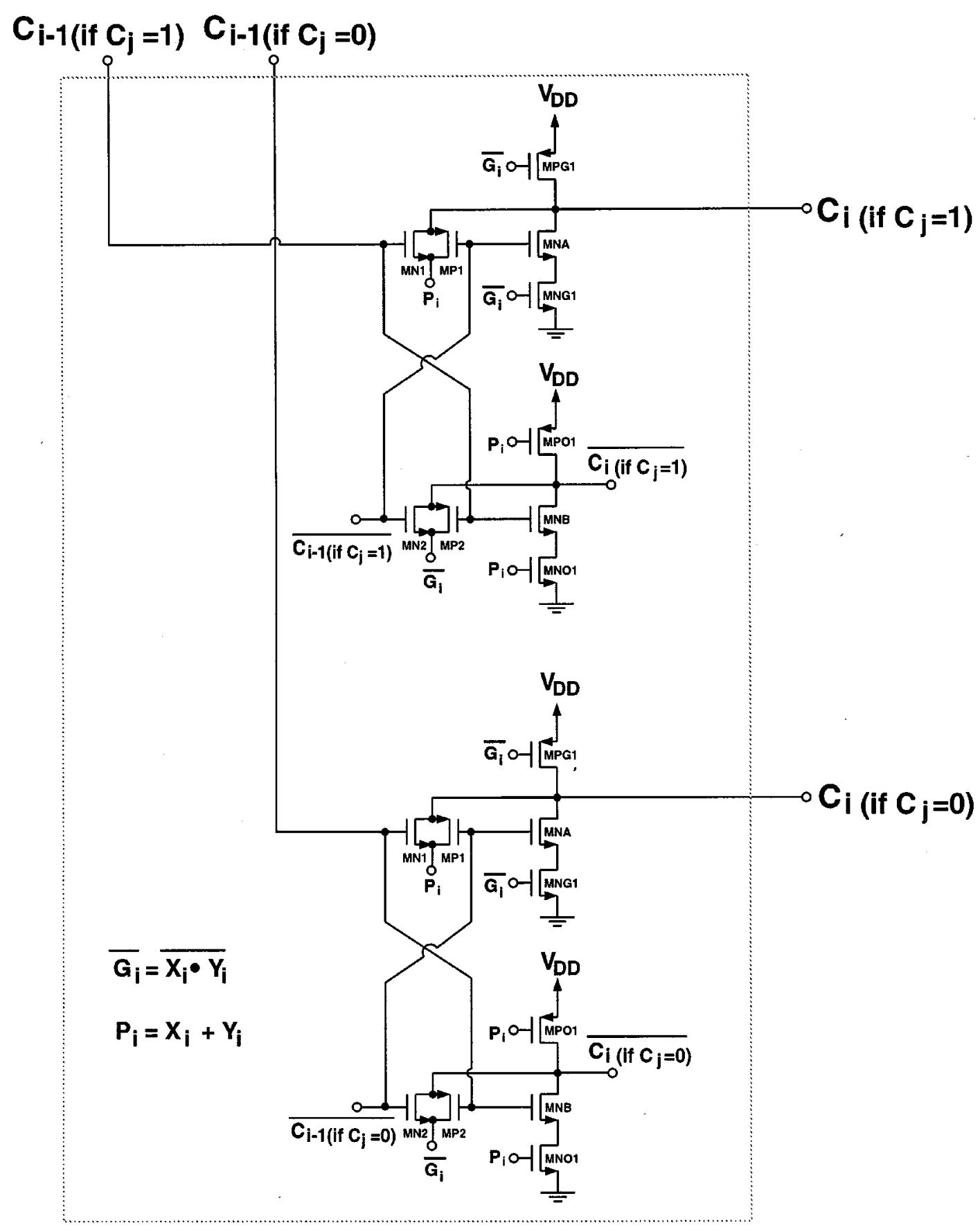

Fig. 3. The 1-bit PTBCS circuit.

adder designed is 16-bit. The SICNB CCS adder is also advantageous for implementing adders with more than 16 bits. The critical path of a 32-bit adder implemented by cascading two conventional 16-bit CCS adders involves 16 stages of logic gates. In contrast, the critical path of a 32-bit adder implemented by cascading two 16-bit SICNB CCS adders involves 12 stages of logic gates, which is 4 stages fewer as compared to the conventional CCS approach. The critical path of a 64-bit adder implemented by cascading four conventional 16-bit CCS adders involves 32 stages of logic gates, which is double as compared to the 32-bit case. On the other hand, the critical path of a 64-bit adder implemented by cascading four SICNB CCS adders, in- volves 24 stages of logic gates, which is only $50 \%$ more as compared to the 32-bit case. Therefore, when the adder is getting bigger, the speed advantages of the SICNB CCS adder over the CCS adder are more noticeable.

\section{CONCLUSION}

In this paper, a CCS adder circuit with an SICNB structure for low-voltage VLSI implementation has been described. Owing to the SICNB structure, the new 16-bit SICNB CCS adder provides a $37 \%$ faster speed as compared to the conventional CCS adder based on the SPICE results. 


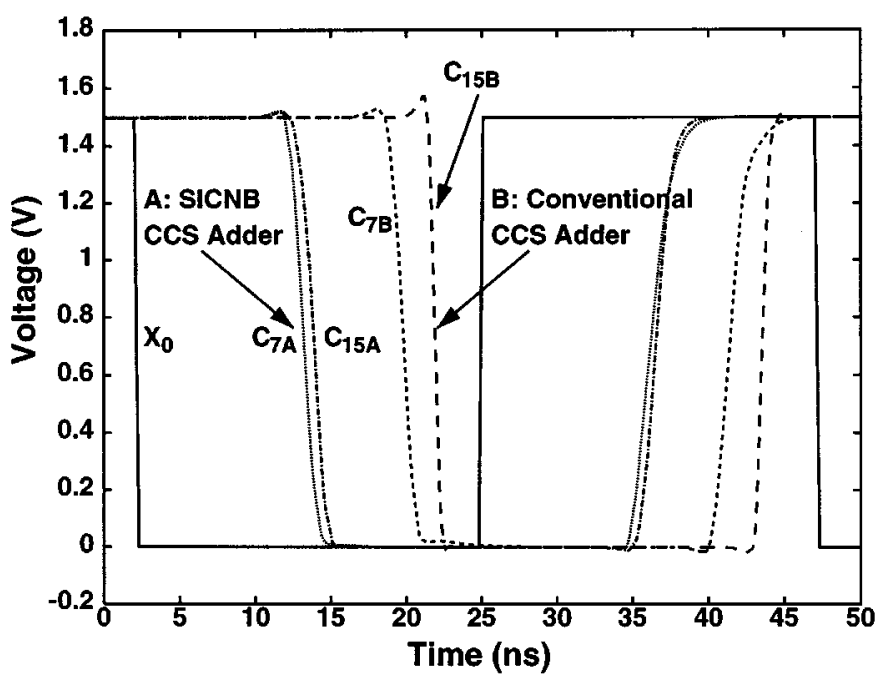

Fig. 4. Transients of the 1.5-V 16-bit SICNB structure CCS circuit and the CSS circuit.

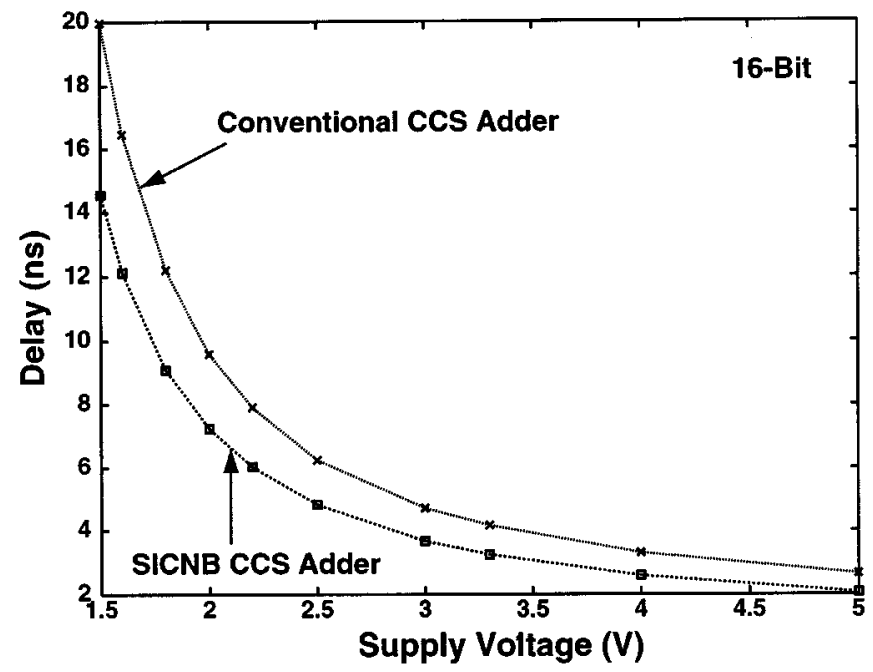

Fig. 5. Propagation delay versus supply voltage of the 16-bit SICNB structure and CCS circuit.

TABLE I

POWER CONSUMPTION OF A 16-BIT ADDER IMPLEMENTED BY CONVENTIONAL CCS TECHNIQUE AND THE SICNB CCS TECHNIQUE

\begin{tabular}{c|c|c}
\hline Supply Voltage & Conventional CCS Adder & SICNB CCS Adder \\
\hline $1.5 \mathrm{~V}$ & $0.22 \mathrm{~mW}$ & $0.39 \mathrm{~mW}$ \\
\hline $2 \mathrm{~V}$ & $0.4 \mathrm{~mW}$ & $0.71 \mathrm{~mW}$ \\
\hline $2.5 \mathrm{~V}$ & $0.65 \mathrm{~mW}$ & $1.13 \mathrm{~mW}$ \\
\hline $3 \mathrm{~V}$ & $0.97 \mathrm{~mW}$ & $1.68 \mathrm{~mW}$ \\
\hline
\end{tabular}

\section{REFERENCES}

[1] M. Suzuki, N. Ohkubo, T. Shinbo, T. Yamanaka, A. Shimizu, K. Sakai, and Y. Nakagome, "A 1.5-ns 32-b CMOS ALU in double pass-transistor logic,” IEEE J. Solid-State Circuits, vol. 28, pp. 1145-1150, Nov. 1993.
[2] N. Ohkubo, M. Suzuki, T. Shinbo, T. Yamanaka, A. Shimizu, K. Sakai, and Y. Nakagome, "A 4.4ns CMOS $54 \times 54-b$ multiplier using pass-transistor multiplexer," IEEE J. Solid-State Circuits, vol. 30, pp. 251-256, Mar. 1995.

[3] C. C. Hung, J. H. Lou, and J. B. Kuo, "A CMOS quasistatic Manchester-like carry-look-ahead circuit," in Low-Voltage CMOS VLSI Circuits, J. B. Kuo and J. H. Lou, Eds. New York: Wiley.

[4] J. B. Kuo and J. H. Lou, Low-Voltage CMOS VLSI Circuits. New York, NY: Wiley, 1999.

[5] I. S. Hwang and A. L. Fisher, "Ultrafast compact 32-bit CMOS adders in multiple-output domino logic," IEEE J. Solid-State Circuits, vol. 24, pp. 358-369, Apr. 1989.

[6] K. Yano, T. Yamanaka, T. Noshida, M. Saito, K. Shimohigashi, and A. Shimizu, "A 3.8-ns CMOS $16 \times$ 16-b multiplier using complementary pass-transistor logic," IEEE J. Solid-State Circuits, vol. 25, pp. 388-394, Apr. 1990.

[7] J. H. Lou and J. B. Kuo, "A 1.5V boostrapped pass-transistor-based carry look-ahead circuit suitable for low-voltage CMOS VLSI," IEEE Trans. Circuits Syst. I, vol. 45, p. 1191-1194, Nov. 1998.

[8] J. B. Kuo, S. S. Chen, C. S. Chiang, K. W. Su, and J. H. Lou, "A 1.5V BiCMOS dynamic logic circuit using a 'BiPMOS pull-down' structure for VLSI implementation of full adders," IEEE Trans. Circuits Syst. I, vol. 41, pp. 329-332, Apr. 1994.

[9] J. B. Kuo and J. H. Lou, Low-Voltage CMOS VLSI Circuits. New York: Wiley, Jan. 1999, pp. 354-355.

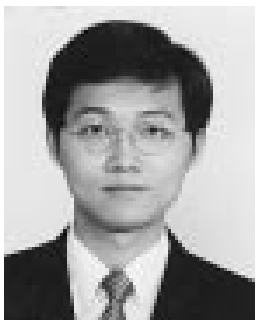

Yen-Mou Huang received the B.S. degree in control engineering from National Chiao Tung University, Hsin-chu, Taiwan, and the M.S.E.E. degree in electrical engineering from National Taiwan University, Taipei, Taiwan, in 1997 and 1999, respectively.

He is currently a Circuit Design Engineer with VIA Technologies, Inc., Taiwan.

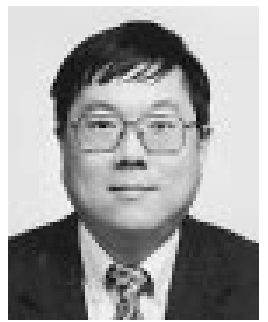

James B. Kuo (F'99) received the B.S.E.E. degree from National Taiwan University (NTUEE), Taipei, Taiwan, in 1977, the M.S.E.E. degree from Ohio State University, Columbus, in 1978, and the Ph.D.E.E. degree from Stanford University, Stanford, CA, in 1985.

He was a Research Engineer during 1978-1981 with Penril Data Communications, Rockville, MD, and Racal Vadic, Sunnyvale, CA, working on integrating telecommunication modem chips using CMOS technology. He then joined the IC Lab, Stanford University, as an Engineering Research Associate, working on BiCMOS devices. Since 1987, he has been with NTUEE. Currently, he is a Professor at the University of Waterloo, Waterloo, ON, Canada, on leave from NTUEE. His research expertise is in the field of low-voltage CMOS VLSI circuits and SPICE compact modeling of deep-submicron bulk and SOI CMOS and BiCMOS VLSI devices. He has published over 100 international journal papers and authored eight books, including Low-Voltage CMOS VLSI Circuits (New York: Wiley, 1999) and CMOS VLSI Engineering: Silicon-On-Insulator (SOI) (Norwell, MA: Kluwer, 1998). He has graduated over 38 M.S. and Ph.D. students specializing in CMOS circuit designs and device modeling, and currently works in leading microelectronic companies in the U.S. and Taiwan.

Dr. Kuo serves as a member of the International Advisory Board of the IEEE Circuits and Devices Magazine and Chair of the Membership Committee for the IEEE Electron Devices Society, for which he is also a Distinguished Lecturer. In 1999, he became an IEEE Fellow for his contributions to modeling CMOS VLSI devices. 